Discussiones Mathematicae

\title{
REGULAR COLORINGS IN REGULAR GRAPHS
}

\author{
ANTON BERNSHTEYN \\ Department of Mathematics, University of Illinois at Urbana-Champaign \\ e-mail: bernsht2@illinois.edu \\ OMID KHORMALI \\ Department of Mathematical Sciences, University of Montana \\ e-mail: omid.khormali@umconnect.umt.edu \\ RYAN R. MARTIN \\ Department of Mathematics, Iowa State University \\ e-mail: rymartin@iastate.edu \\ JoNATHAN ROLLiN \\ Department of Mathematics, Karlsruhe Institute of Technology \\ e-mail: jonathan.rollin@kit.edu \\ DANNY RORABAUGH \\ Department of Mathematics and Statistics, Queen's University \\ e-mail: rorabaug@email.sc.edu \\ SONGLing SHAN \\ Department of Mathematics, Vanderbilt University \\ e-mail: songling.shan@vanderbilt.edu
}

AND

ANDREW J. UzzelL

Mathematics and Statistics Department, Grinnell College

e-mail: uzzellan@grinnell.edu 


\begin{abstract}
An $(r-1,1)$-coloring of an $r$-regular graph $G$ is an edge coloring (with arbitrarily many colors) such that each vertex is incident to $r-1$ edges of one color and 1 edge of a different color. In this paper, we completely characterize all 4-regular pseudographs (graphs that may contain parallel edges and loops) which do not have a $(3,1)$-coloring. Also, for each $r \geq 6$ we construct graphs that are not $(r-1,1)$-colorable and, more generally, are not $(r-t, t)$-colorable for small $t$.
\end{abstract}

Keywords: edge coloring, graph factors, regular graphs.

2010 Mathematics Subject Classification: 05C15.

\title{
REFERENCES
}

[1] S. Akbari and M. Kano, $\{k, r-k\}$-factors of $r$-regular graphs, Graphs Combin. 30 (2014) 821-826. doi:10.1007/s00373-013-1324-x

[2] J. Akiyama and M. Kano, Factors and Factorizations of Graphs: Proof Techniques in Factor Theory (Springer, Heidelberg, 2011). doi:10.1007/978-3-642-21919-1

[3] M. Axenovich and J. Rollin, Brooks type results for conflict-free colorings and $\{a, b\}$ factors in graphs, Discrete Math. 338 (2015) 2295-2301. doi:10.1016/j.disc.2015.05.020

[4] A.Y. Bernshteyn, 3-regular subgraphs and (3,1)-colorings of 4-regular pseudographs, J. Appl. Ind. Math. 8 (2014) 458-466. doi:10.1134/S1990478914040024

[5] B. Bollobás, A. Saito and N.C. Wormald, Regular factors of regular graphs, J. Graph Theory 9 (1985) 97-103. doi:10.1002/jgt.3190090107

[6] J.A. Bondy and U.S.R. Murty, Graph Theory with Applications (American Elsevier Publishing Co., New York, 1976).

[7] F.R.K. Chung and R.L. Graham, Recent results in graph decompositions, in: Combinatorics Proc. 8th British Combinatorial Conference (Swansea, 1981), London Math. Soc. Lecture Note Ser. 52 (1981) 103-123.

[8] L. Lovász, The factorization of graphs. II, Acta Math. Hungar. 23 (1972) 223-246. doi:10.1007/BF01889919

[9] H. Lu, D.G.L. Wang and Q. Yu, On the existence of general factors in regular graphs, SIAM J. Discrete Math. 27 (2013) 1862-1869. doi:10.1137/120895792

[10] J. Petersen, Die Theorie der regulären graphs, Acta Math. 15 (1891) 191-220. doi:10.1007/BF02392606 
[11] M.D. Plummer, Graph factors and factorization: 1985-2003: A survey, Discrete Math. 307 (2007) 791-821. doi:10.1016/j.disc.2005.11.059

[12] V.A. Tashkinov, 3-regular parts of 4-regular graphs, Mat. Zametki 36 (1984) 239-259.

[13] V.A. Tashkinov, Regular parts of regular pseudographs, Mat. Zametki 43 (1988) 263-275.

[14] W.T. Tutte, The subgraph problem, Ann. Discrete Math. 3 (1978) 289-295. doi:10.1016/S0167-5060(08)70514-4

Received 4 October 2017

Revised 7 May 2018

Accepted 10 May 2018 